Fecha de recepción: febrero 2019 Fecha de aceptación: julio 2019 Versión final: noviembre 2020

\section{Possibilidades da Tecnologia Touchscreen para desenvolvimento motor e inclusão digital de pessoas com Paralisia Cerebral}

Carolina S. M. Tavares ${ }^{1}$, Bruna R. Machado², Bruna

M. Bischoff ${ }^{3}$ e Murilo Scoz ${ }^{4}$

\begin{abstract}
Resumo: O presente artigo visa investigar as possibilidades de dispositivos touchscreen para o estímulo motor de pessoas com Paralisia Cerebral (PC) com intuito de contribuir para acessibilidade de objetos de design ao maior número de usuários conforme a premissa do Design Universal. Os procedimentos metodológicos empregados foram revisão bibliográfica acerca das tecnologias touchscreen e terapias para pessoas com Paralisia Cerebral e entrevistas semi estruturadas com profissionais da área de fisiatria, fisioterapia, fonoaudiologia, terapia ocupacional e educação física com experiência no atendimento ao público em questão, atuantes no Centro Catarinense de Reabilitação (CCR) de Florianópolis, Santa Catarina, no Brasil. Pela observação dos aspectos analisados, concluiu-se que os dispositivos touchscreens se apresentam como uma ferramenta a ser aprimorada, visto que possibilitam uma variedade de gestos manuais superior à outros dispositivos de tecnologia assistiva computacional. O tamanho e a interface das telas sensíveis ao toque foram alguns dos principais obstáculos identificados na utilização de tais tecnologias por pacientes com Paralisia Cerebral. É relevante, portanto, no campo do Design Universal, explorar o cruzamento entre níveis de acometimento motor de pessoas com PC e os gestos possíveis nas telas, de modo a contribuir para o avanço da acessibilidade nos dispositivos touchscreen não somente às pessoas com essa disfunção neurológica, mas também às diversas deficiências motoras correlatas.
\end{abstract}

Palavras chave: touchscreen - Paralisia Cerebral - Design Universal.

[Resumos em espanhol e inglês nas páginas 203-204]

(1) Programa de Pós-Graduação em Design, Universidade do Estado de Santa Catarina (UDESC), Brasil.

(2) Programa de Pós-Graduação em Design de Vestuário e Moda, Universidade do Estado de Santa Catarina (UDESC), Brasil.

(3) Programa de Pós-Graduação em Design, Universidade do Estado de Santa Catarina (UDESC), Brasil.

(4) Programa de Pós-Graduação em Design, Universidade do Estado de Santa Catarina (UDESC), Brasil. 


\section{Introdução}

Com o desenvolvimento de dispositivos digitais móveis, o acesso à tecnologia se aproxima da população mundial. Em meio a este rápido avanço tecnológico, é relevante considerar a inclusão digital de pessoas com deficiência, fator primordial para garantir, além do acesso à tecnologia, o convívio e interação social deste público. Desta forma, o presente artigo objetiva investigar as possibilidades de dispositivos touchscreen para o estímulo motor de pessoas com paralisia cerebral.

O estudo realizado se dividiu em três fases: primeiramente foi realizada uma revisão bibliográfica, de modo a reunir o referencial teórico sobre temas como Design Universal, inclusão digital, paralisia cerebral, suas classificações e novas tecnologias, com enfoque nos aparelhos de tela sensível ao toque; e posteriormente sucederam-se entrevistas com profissionais do Centro Catarinense de Reabilitação (CCR), que atendem diariamente pacientes com paralisia cerebral, principalmente na ala infantil.

De acordo com informações da World Health Organization (2012), a reabilitação, processo que visa a melhoria da funcionalidade do indivíduo com o ambiente, atua diretamente no desenvolvimento de crianças com paralisia cerebral, estabilizando-os para a vida em sociedade e estimulando sua independência motora e psicológica. O processo de reabilitação geralmente acontece por um período determinado de tempo e reduz o impacto de diversas condições de saúde.

Após o levantamento bibliográfico, entrevistas realizadas com profissionais das áreas de fisiatria, fisioterapia, terapia ocupacional e fonoaudiologia foram valiosas para a compreensão sobre as rotinas de crianças com paralisia cerebral e validação de hipóteses acerca das possibilidades e dificuldades de manuseio, acionamento e interação por parte dos pacientes. A partir deste contexto, observou-se a necessidade de explanar os atuais usos e limitações de aparelhos touchscreen, em seus diversos formatos, como ferramenta a ser aprimorada para o estímulo motor de pessoas com paralisia cerebral e, consequentemente, das demais disfunções motoras. Com a compreensão de que a paralisia cerebral pode ser classificada em escalas, baseadas em cinco níveis de acordo com o acometimento motor, vê-se a importância de desenvolver interfaces que considerem os movimentos possíveis nestes níveis, facilitando a navegação, atuando com o estímulo necessário a cada usuário e que se mantenham atrativas, com aspectos lúdicos.

\section{Referencial Teórico}

Com o desenvolvimento de dispositivos digitais móveis, o acesso à tecnologia se aproxima da população mundial. Ao considerar que aproximadamente 45 milhões de pessoas (ou o equivalente a $23,9 \%$ da população brasileira) declaram ter alguma deficiência (Censo demográfico do Instituto Brasileiro de Geografia e Estatística, realizado em 2010), a acessibilidade digital se apresenta como fator contribuinte para o desenvolvimento social, motor e intelectual dessas pessoas na atualidade. É necessário realizar estudos sobre a maneira como pessoas com deficiência interagem com objetos tecnológicos e compreendem as informações por eles transmitidas, de modo a incentivar a inclusão social. Observa-se, 
portanto, o crescimento de campos de estudo como o Design Universal, desenvolvido na década de 90 principalmente por profissionais das áreas de Design e Arquitetura, na Universidade da Carolina do Norte (EUA), e o Design Inclusivo que objetivam o projeto de produtos e ambientes acessíveis a todos, independentemente de possíveis restrições físicas, sociais, ou econômicas, e fomentam inclusão de pessoas das mais diversas idades, características pessoais e habilidades (Carletto, Cambiaghi, 2008). A Classificação Internacional de Funcionamento, Incapacidade e Saúde (CIF), categorização atual da Organização Mundial de Saúde relacionada à deficiência, baseada em um modelo social, no qual a deficiência é "encarada como uma situação de desajuste, por vezes existente, entre as capacidades humanas e características do meio" (Simões, Bispo, 2006, p. 28), reforça a premissa de que o objeto ou ambiente deve fornecer os elementos, ferramentas e instruções necessários ao uso por todos.

Entre as deficiências infantis, estima-se que a incidência da paralisia cerebral (PC) seja de 2 a 3 por 1.000 bebês nato-vivos em países desenvolvidos, segundo dados da SCPE (Surveillance of Cerebral Palsy in Europe), projeto de pesquisa e registros sobre paralisia cerebral fundado pelo European Union Health Programme. Em países subdesenvolvidos, como o Brasil, a estimativa chega a 7 em cada 1000, sendo a causa mais frequente de deficiência motora na infância. "A explicação para a diferença na magnitude da prevalência entre estes dois grupos de países é atribuída às más condições de cuidados pré-natais e ao atendimento primário às gestantes" (Brasil, 2014, p. 13). Considerando que os quadros paralisia cerebral são permanentes mas não inalteráveis, tornam-se relevantes as pesquisas acerca de tecnologias e meios para a reabilitação, acessibilidade e estímulo da autonomia destas pessoas. Suas lesões podem afetar as funções motoras, cognitivas, a visão, a audição e ocasionar epilepsia.

A necessidade de agrupamento e classificação da paralisia cerebral se dá pelo alto grau de variabilidade entre casos e, por serem mais frequentes, as disfunções motoras conferem suporte para tais categorizações. As classificações focam na identificação do nível de comprometimento motor das funções motoras globais, aplicando o Sistema de Classificação da Função Motora Grossa (GMFCS), e de função dos membros superiores, com escalas como o Sistema de Classificação das Capacidades de Manipulação (MACS) e o Sistema de Classificação da Função Motora Bimanual Fina (BFMF) (Brasil, 2014; SCPE, 2018). As escalas aqui apresentadas proporcionam a classificação da criança ou adolescente com PC em cinco níveis, sendo o primeiro composto por limitações mais brandas e o quinto por restrições mais graves. Cada nível destes sistemas classificatórios pressupõem competências específicas acerca dos indivíduos, possibilitando, portanto, uma quantidade expressiva ou reduzida de movimentos possíveis.

O Gross Motor Function Classification System (GMFCS), muito utilizado nas práticas clínicas, nos planejamentos de reabilitação e nas pesquisas, baseia-se nos movimentos voluntários, com foco no sentar, transferências e mobilidade. A classificação, que avalia a mobilidade funcional, engloba a faixa etária de 0 a 12 anos, com subdivisões a cada 2 anos. Atualmente foi publicada uma nova versão desta classificação, adicionando a faixa de 12 a 18 anos. O Bimanual Fine Motor Function (BFMF) é um sistema de pontuação para a motricidade fina que considera o envolvimento simétrico ou assimétrico dos membros superiores, verificando as funções de cada mão especificamente. Já o Manual Ability 
Classification System (MACS), uma escala recente, consiste em um sistema que foca no desempenho bimanual de atividades da vida diária da criança em casa, na escola, ou na comunidade com objetos do seu alcance. Compreende uma faixa etária de 4 a 18 anos. $\mathrm{O}$ MACS avalia a habilidade global dos usuários na manipulação de objetos, sem analisar a função de cada mão separadamente. Esta é a principal diferença do MACS com relação ao BFMF, que avalia os membros individualmente (Brasil, 2014; SCPE, 2018).

$\mathrm{O}$ uso de artefatos e aparelhos, digitais ou não, nas terapias de reabilitação é comum e benéfico para a estimulação do paciente. Para as pessoas com deficiência, em especial a paralisia cerebral, a acessibilidade e interação digital se dão principalmente por adaptações e tecnologias assistivas, que "são recursos e serviços que visam promover a funcionalidade e a autonomia da pessoa com paralisia cerebral, minimizando os problemas e as dificuldades decorrentes dessa condição de saúde" (Brasil, 2014, p. 45). Atualmente existem diversas propostas para criação e avaliação da usabilidade digital e, mais especificamente, da web, porém percebe-se a falta de padronização, principalmente quando a acessibilidade é levada em consideração. O documento Web Content Accessibility Guidelines (WCAG) 2.0, ou Diretrizes de Acessibilidade para Conteúdo Web, por exemplo, define maneiras de tornar o conteúdo online mais acessível através de quatro princípios: perceptível, operável, compreensível e robusto (Bassani et al., 2010). Observa-se que estas premissas são igualmente válidas aos diversos dispositivos de tecnologia assistiva, indo além dos conteúdos web.

Em meio às novas tecnologias, destacam-se os dispositivos touchscreen que, pela portabilidade e fácil acesso à informação, têm se tornado cada vez mais familiares à população com os smartphones e tablets. Mesmo não sendo elaborados como tecnologias assistivas inicialmente, se adaptam e podem apresentar recursos de estímulo manual com o toque na tela e interfaces específicas. Segundo Ribeiro (como citado em Shneiderman \& Plaisant, 2014, p. 4), "os touchscreens são muitas vezes integrados em apps para utilizadores inexperientes nos quais o teclado deixa de existir e o toque passa a ser a principal forma de interação". Observando que as disfunções motoras são presentes na maioria dos casos de crianças com paralisia cerebral, vê-se nos aparelhos touchscreen uma possibilidade de estímulo para a movimentação dos membros superiores e, mais precisamente, para as mãos, uma vez que diversos movimentos são possíveis na tela.

\section{Procedimentos Metodológicos}

A natureza da presente pesquisa é qualitativa e seus resultados se baseiam na utilização de estudos exploratórios como entrevistas e visitas a campo. Este trabalho tem como hipótese o entendimento de que a utilização de aparatos de tecnologia touchscreen pode colaborar com o tratamento de acometimentos motores relacionados à paralisia cerebral. O objetivo geral, portanto, é esclarecer a importância potencial deste tipo de tecnologia no âmbito do tratamento da PC, e os objetivos específicos consistem em realizar entrevistas com profissionais da saúde, buscar o estado da arte dos temas adjacentes na literatura apropriada e compreender as limitações e possibilidades no uso de tecnologias touchscreen na reabilitação de paralisia cerebral. 
Esta pesquisa teve início com uma revisão bibliográfica para embasar o referencial dos eixos teóricos. Em seguida, entrevistas semiestruturadas com profissionais da área e visitas a instituição de saúde foram as ferramentas utilizadas para colaborar com os achados na literatura. Para o perfil dos entrevistados recorreu-se ao Centro Catarinense de Reabilitação, órgão com grande representatividade em relação a programas de reabilitação para pacientes com paralisia cerebral e outras disfunções neurológicas no estado de Santa Catarina, selecionando assim os profissionais que atuam com maior frequência e impacto na reabilitação destes pacientes que se concentram nas áreas de fisiatria, fisioterapia, fonoaudiologia e terapia ocupacional.

Foram coletados dados quantitativos em relação ao centro bem como informações qualitativas sobre os procedimentos terapêuticos empregados. As entrevistas aconteceram em janeiro do ano 2018, e a primeira delas foi com a médica fisiatra que também ocupa o cargo da gerência do CCR. As perguntas realizadas versavam sobre dados gerais do atendimento oferecido pelo Centro como: quantidade de pacientes atendidos; dentre estes, a porcentagem de acometidos por PC; duração média dos tratamentos; dispositivos terapêuticos e objetos utilizados, sendo tecnológicos ou não; e tipos principais de movimentos desenvolvidos.

As demais entrevistas se realizaram na seguinte ordem: terapeuta ocupacional juntamente com educador físico, fisioterapeuta e fonoaudiólogo. Os questionamentos foram similares para todos estes profissionais, porém buscando informações específicas de cada uma das especialidades em referência a: principais metas na reabilitação de pacientes com PC; vivência prática da realidade do tratamento dentro do Centro e também no convívio familiar; utilização das escalas MACS e GMFCS; e principalmente, se aparelhos de tecnologia touchscreen são ou podem vir a ser utilizados de forma a apoiar o tratamento realizado. Todas as reuniões contaram com três entrevistadores. A partir da transcrição integral das gravações e da leitura do material, realizou-se a análise dos dados.

Neste trabalho, primeiramente são demonstrados os conceitos gerais sobre os temas Design Universal, paralisia cerebral, novas tecnologias e dispositivos touchscreen em tratamentos e terapias relacionadas à saúde, além das principais escalas de avaliação da PC: MACS e GMFCS. Em seguida, a metodologia utilizada é abordada e é apresentado o CCR, assim como os profissionais de saúde que lá trabalham e se dispuseram a serem entrevistados, e suas respectivas especialidades. Na sequência as entrevistas com os profissionais são descritas e há uma discussão no qual os temas citados são relacionados com a fundamentação teórica apresentada anteriormente. E, finalmente, se apresentam as considerações finais juntamente com sugestões para futuras pesquisas.

\section{O Centro Catarinense de Reabilitação}

O Centro Catarinense de Reabilitação (CCR), localizado em Florianópolis - SC é uma instituição pública habilitada pelo Ministério da Saúde em Centro Especializado em Reabilitação Física e Intelectual pertencente à Secretaria de Estado da Saúde de Santa Catarina. Os programas de reabilitação realizados pelo centro são para pacientes adultos e infantis que apresentam disfunções neurológicas com sequelas motoras e funcionais, e tam- 
bém sequelas ligadas a deficiência intelectual. Dentre as disfunções atendidas estão a Paralisia Cerebral (PC), Esclerose Lateral Amiotrófica (ELA), Doença de Parkinson (DP), Mielomeningocele, Lesão encefálica, Lesão medular, Transtorno do espectro do autismo, Síndrome de Down, entre outros. Na reabilitação, as seguintes áreas de atendimento são compreendidas pelos programas: deficiência física (Programa de Neuroreabilitação Adulto; Programa de Reabilitação Pediátrica; Atenção às Pessoas Ostomizadas) e deficiência intelectual e espectro do autismo (Habilitação e Reabilitação Intelectual; Reabilitação do Espectro do Autismo ${ }^{1}$ ).

Para o tratamento dos pacientes, o CCR opera com uma equipe multidisciplinar. Dentre os profissionais atuantes estão médicos fisiatras, médicos ortopedistas, médicos neurologistas, assistentes sociais, psicólogos, nutricionistas, terapeutas ocupacionais, fonoaudiólogos, fisioterapeutas, entre outros. A partir de uma junta com o corpo clínico do centro são identificados os problemas e necessidades que impossibilitam o paciente de manter uma funcionalidade ideal na interação com seu entorno e, dessa forma, são estabelecidas medidas que viabilizem os indivíduos a atingirem a independência e autodeterminação. Sendo assim, os programas variam quanto ao tempo de duração, bem como as áreas desenvolvidas durante o seu curso, pois estes são determinados conforme a disfunção apresentada pelo paciente e do seu grau de acometimento motor e intelectual. O CCR possui dois tipos de atendimentos: consultas pontuais, que em média somam trezentas consultas semanais; e os programas de reabilitação, que atualmente atendem entre cento e sessenta a cento e setenta pacientes. Nesta pesquisa, os entrevistados fazem parte da equipe de saúde do centro e atuam com grande frequência no tratamento dos pacientes de paralisia cerebral. Dentre os profissionais que colaboraram, está a gerente do centro, presente na instituição desde 2004. Médica com especialização em Medicina Física e Reabilitação e Mestre em Neurociências, atua principalmente na Reabilitação Pediátrica, Reabilitação Neurológica, Toxina botulínica e Tratamento da dor, também é Membro da Academia Americana de Paralisia Cerebral e Medicina do Desenvolvimento (AACPDM) desde 2014. Os outros entrevistados são profissionais das áreas de Terapia Ocupacional (TO), Fisioterapia, Educação Física e Fonoaudiologia, serão mantidos em anonimato.

\section{Resultados e Discussão}

Nas entrevistas realizadas com os profissionais do corpo clínico da ala infantil do CCR, foi possível observar as rotinas, procedimentos, expectativas e limitações enfrentadas, de modo a angariar conhecimentos acerca da reabilitação pediátrica da paralisia cerebral. Foi recorrente o apontamento de que a limitação financeira e de recursos do centro resulta no uso reduzido de dispositivos tecnológicos nos programas de reabilitação realizados pela instituição. Contudo, este não é um entrave no desenvolvimento das terapias atualmente, mas sim uma possibilidade de melhoria e ampliação de ferramentas que auxiliem os pacientes e suas famílias.

De acordo com os entrevistados, o centro faz uso de escalas para classificação dos pacientes com PC, servindo de norteamento para os objetivos desenvolvidos especialmente em cada caso. Além da GMFCS e da MACS, apresentadas anteriormente neste artigo, também 
é utilizado o Sistema de Classificação da Função de Comunicação (CFCS), que analisa o desempenho da comunicação diária do paciente. Reforça-se o que uso da GMFCS é mais frequente que a MACS, uma vez que, ao classificar a criança pela função motora grossa, o resultado da função dos membros superiores e, consequentemente, da MACS é similar. Para definir as classificações, os terapeutas aplicam o Gross Motor Function Measure (GMFM) que, através de escores em diversos itens, resultam em uma pontuação. O CCR faz uso da versão reduzida do GMFM, contendo 66 itens com escores de 0 a 3, divididos em cinco macrocategorias: deitar e rolar; sentar; engatinhar e ajoelhar; em pé; andar, correr e pular. $\mathrm{O}$ fisioterapeuta elucidou que até os quatro anos o paciente pode mudar de nível na classificação, mas que após essa idade a mudança se torna mais difícil, uma vez que os quadros se distanciam e seria necessário adicionar ou subtrair uma média de 40 pontos da GMFM-66, conforme mostrado no quadro abaixo. Desta forma, aos quatro anos a criança já terá sua classificação fixa, podendo melhorar suas funções em questão de qualidade, se bem estimulada, porém dificilmente conseguirá adquirir novas habilidades.

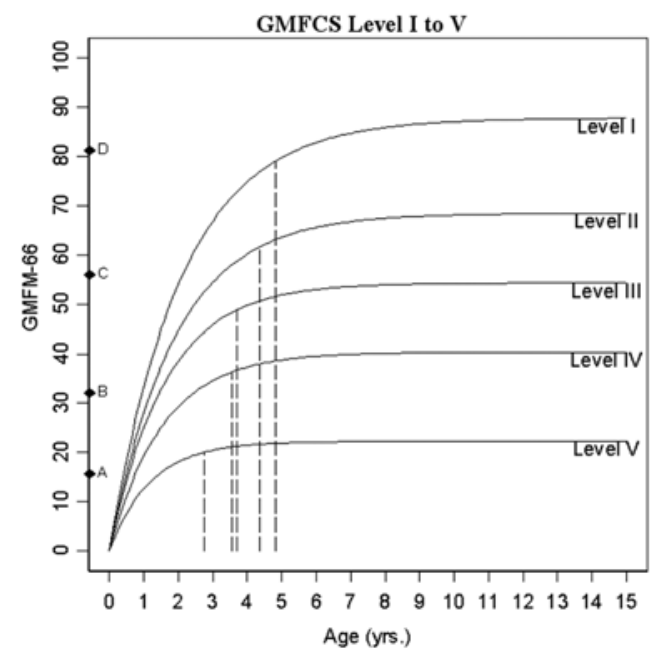

Figura 1. Gráfico de relação entre idade e pontuação no teste GMFM-66, resultando nos níveis GMFCS. Fonte: blyum.typepad.com, acessado em 20/01/2018.

Dentre os aparatos tecnológicos apontados como possibilidades vantajosas a serem incluídos no trabalho realizado pelos entrevistados estão o uso do video game Wii, projetores, tablets, mesas touchscreen e vocalizadores. Também observa-se a busca por aparelhos novos e tecnológicos ligados diretamente às terapias, como a esteira suspensa, andadores e exo- 
esqueletos, no caso da fisioterapia. O video game Wii foi citado pelo fisioterapeuta como uma alternativa interessante nos tratamentos, trabalhando com a gameterapia. Reforça-se que atividades lúdicas que envolvam jogos prendem a atenção da criança e criam objetivos dentro das atividades terapêuticas realizadas. O centro tem planos para implementá-lo no futuro. Outros video games foram mencionados, como o Xbox e o Play Station 4, porém não seriam as primeiras opções de compra para uso terapêutico no centro, uma vez que o Play Station 4 é mais caro que o Wii e que o Xbox escaneia todo o corpo com seu sensor de movimento. Como o terapeuta teria que, muitas vezes, auxiliar o paciente a realizar determinado movimento, esse escaneamento poderia confundir as silhuetas. O Wii, com controle, resolveria esta situação.

Tablets também foram citados como opções para o estímulo motor. Os smartphones, pelo tamanho de tela, não foram indicados. Atualmente, os dispositivos touchscreen são utilizados no centro principalmente na área de fonoaudiologia com a Comunicação Alternativa, que designa um conjunto de procedimentos direcionados a pessoas acometidas por alguma doença, deficiência, ou outra situação momentânea que impede a comunicação por meio de recursos geralmente utilizados, como a fala (MANZINI, 2006), mas são considerados para jogos e estimulações cognitivas. Os entrevistados ressaltaram que estes dispositivos não são essenciais para as terapias, mas são eficazes para chamar a atenção da criança para determinado exercício, complementando as demais atividades. Um dos exemplos de sua aplicação é dado pelo fisioterapeuta, que explica que ao solicitar que o paciente vá de uma ponta a outra da sala, buscando trabalhar a locomoção, ele não irá realizar a movimentação se não tiver um objetivo. Se um tablet ou outro aparelho similar for colocado na ponta da sala, a criança terá interesse em se deslocar até lá, onde poderá realizar uma tarefa na tela ou jogar parte de um jogo. O dispositivo pode, portanto, funcionar como um atrativo e, ao mesmo tempo, estimular o movimento manual e a cognição dependendo da tarefa realizada na tela. A combinação de terapias, aparelhos e brincadeiras é importante para o desenvolvimento da criança com PC. O centro não possui tablets atualmente e quando utilizados, são trazidos pela criança.

Outros dispositivos touchscreen foram citados, como mesas digitais e interativas. A Playtable, é um exemplo destes objetos, desenvolvida no estado de Santa Catarina com tecnologia infrared, que permite a acessibilidade de crianças com deficiência motora e psíquica. A empresa centraliza diversos jogos e aplicativos, focando nos estímulos motores, cognitivos, emocionais, escolares e sociais.

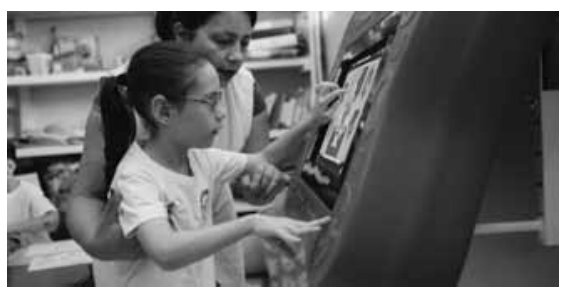

Figura 2. Criança utilizando a Playtable com auxílio de profissional. Fonte: panoramadenegocios.com.br, acessado em 22/01/2018. 
A participação familiar foi considerada crucial no processo de reabilitação dos pacientes com PC e outras disfunções. As sessões de fisioterapia, fonoaudiologia, terapia ocupacional e educação física, realizadas no centro, são semanais e duram entre 30 a 50 minutos, dependendo do desempenho e idade do paciente, portanto a repetição de alguns exercícios das sessões e os estímulos recebidos em casa e na escola impactam consideravelmente no desempenho destes pacientes. Conforme relatado pela gerência, todos que estão em idade escolar são incentivados a frequentá-la. O CCR oferece orientação para os professores para que recebam estes alunos de forma adequada. Entretanto os profissionais de saúde apontaram algumas dificuldades na colaboração familiar, muitas vezes por negação do quadro clínico da criança e outras vezes pela demanda profissional ou por compromissos domésticos. Consequentemente, estímulos nos quais o paciente tenha autonomia para cumprir sozinho se fazem relevantes, como no caso de jogos educativos em dispositivos eletrônicos, que se apresentam como um possível complemento no processo de reabilitação.

O profissional de fonoaudiologia frisou a importância de aproximar as ferramentas, objetos e exercícios utilizados nos programas de reabilitação com a realidade da criança. Sendo assim, uma possibilidade que se torna pertinente para o desenvolvimento de aplicativos e programas é projetá-los de forma customizável, nos quais seja permitido alterar imagens e palavras para que se relacionem com a rotina do paciente. No caso das metodologias para a Comunicação Alternativa, são utilizadas fotos dos locais que a criança frequenta, os nomes de animais que ela tem contato e outras informações que fazem parte do seu repertório social. Compreende-se que esta abordagem aponta ser produtiva para desenvolvimento de jogos e outros programas educativos direcionados para os pacientes com paralisia cerebral.

A partir das entrevistas, constatou-se que o uso terapêutico de tecnologias touchscreen deve levar em consideração a classificação nas escalas motoras e de comunicação. Uma das hipóteses levantadas é a de maior aplicabilidade desta ferramenta para pacientes dos níveis I, II e III do GMFCS, uma vez que estes níveis conseguem ter maior controle sobre a motricidade geral do corpo, podendo receber estímulos manual e fino. Ainda assim, o tamanho de botões e a sensibilidade das telas se apresentam como elementos a serem repensados em projetos gráficos para este público, pois impactam na adoção destas tecnologias. Os pacientes com diagnósticos mais graves, de níveis IV e V, apresentam um comprometimento motor mais avançado para o uso de telas sensíveis ao toque, o que torna a precisão dos movimentos difícil, fazendo com que a criança selecione botões, setas ou outros direcionamentos erroneamente com frequência ou mesmo nem conseguindo selecioná-los.

Outro entrave citado no uso de jogos eletrônicos e programas é a quantidade de estímulos que são encontrados na tela. A dificuldade de concentração, em virtude da idade e da disfunção neurológica, é um obstáculo no tratamento terapêutico destes pacientes. Possibilidades de estímulo exacerbadas não desempenham bem para este público, devendo o objetivo de cada atividade ser simplificado e claro. Se tratando de jogos eletrônicos, entende-se que as opções de navegação também devam seguir esta mesma lógica. 


\section{Conclusões}

A partir da pesquisa bibliográfica e das entrevistas realizadas com os profissionais da saúde que atendem no CCR, pôde-se verificar a importância potencial dos dispositivos touchscreen como complemento no tratamento de acometimentos relacionados à PC, tanto em sessões terapêuticas como para estímulos contínuos, realizados pela escola e pela família. Concluiu-se que tais aparatos se apresentam como uma ferramenta útil e que pode ser aprimorada. Seu uso se dá atualmente, de uma forma geral, relacionado à área da fonoaudiologia buscando promover movimentos referentes à comunicação, porém existe um amplo campo de possibilidades a ser explorado no que tange a reabilitação de pessoas com paralisia cerebral, visto que possibilitam uma variedade de gestos manuais superior a outros dispositivos de tecnologia assistiva computacional, como o teclado, que exige precisão, especificidade e limitada variação de movimentos para o acionamento das teclas. As dificuldades enfrentadas por pessoas com paralisia cerebral na interação com dispositivos tecnológicos e no desempenho dos movimentos manuais finos, ocasionadas principalmente pelas disfunções motoras, devem ser analisadas e amenizadas através de projetos gráficos que levem em consideração a especificidade deste público. Identificou-se que esse limitante impacta no tamanho da tela e também no seu nível de sensibilidade. A dificuldade na precisão dos movimentos dos usuários com PC interfere na usabilidade dos aplicativos, uma vez que suas interfaces apresentam muitos itens clicáveis e áreas pequenas para realizar o toque. A combinação de telas pequenas com o grande aglomerado de elementos pode induzir ao acionamento errado.

Compreende-se, portanto, que para utilizar dispositivos móveis touchscreen, como smartphones e tablets, indica-se que a quantidade de funções, seja com botões, textos ou ícones, seja limitada e que cada elemento seja distante entre si. Buscando aprimorar a interação, a limitação de ações disponíveis por tela é necessária, aumentando a proporção de cada botão clicável e espaçamento entre funções. A simplicidade nas interações das telas é primordial, uma vez que é necessário considerar a possível variação cognitiva dos pacientes, que interfere na usabilidade de dispositivos tecnológicos. O toque pressionado para o acionamento de botões e funções surge como uma possibilidade a ser explorada para minimizar as dificuldades relatadas, pois dificulta que um movimento distônico faça um clique errado.

Embora o tamanho da tela do dispositivo seja relevante na aplicabilidade do objeto para a reabilitação, não seria necessária uma adaptação do objeto em si, como acontece com o computador, que requer mouse adaptado ou um acionador. Dependendo das aplicações em sua interface, uma tela menor, como a dos smartphones, pode ser utilizada. Opções maiores, como a Playtable, mesa digital, são relevantes para interações mais complexas, embora tenham um custo superior.

$\mathrm{O}$ uso de escalas de acometimento motor fino que localizam o paciente quanto as suas capacidades, também surge como um possível norteador para projetos gráficos. A partir do conhecimento dos diferentes níveis da escala, pode-se determinar quais movimentos serão mais produtivos e aplicáveis para cada paciente. Foi identificado que pacientes dos níveis I, II e III do GMFCS, provavelmente tenham capacidade e poderão se beneficiar do uso deste tipo de tecnologia, entretanto para níveis mais agudos de acometimento motor, a tela sensível ao toque não se consolida como a alternativa terapêutica produtiva. 
Apoiado nestas considerações, como sugestão para futuras pesquisas, propõe-se realizar testes de interação com os dispositivos touchscreen por pessoas com PC, de modo a reunir informações quantitativas e qualitativas para a obtenção da correlação entre gestos possíveis e níveis de disfunção motora em casos de PC, contribuindo para o desenvolvimento de interfaces específicas para este público. A partir deste cruzamento, será possível elencar parâmetros para o projeto de interfaces digitais que englobem apenas os gestos possíveis e facilite a usabilidade destes aparelhos.

\section{Notas}

1. Secretária de Estado da Saúde de Santa Catarina. Disponível em: http://portalses.saude. sc.gov.br/index.php?option $=$ com_content $\&$ view $=$ article $\&$ id $=2166 \% 3$ Aprogramas $\&$ catid $=814 \&$ Itemid $=448$. Acesso em 1 nov. 2017

\section{Bibliografia}

Bassani, P.; Behar, P.; Heidrich, R.; Bittencourt, A. \& Ortiz, E. (2010). Usabilidade e acessibilidade no desenvolvimento de interfaces para ambientes de educação à distância. Revista Novas Tecnologias Na Educação, 8(1). http://dx.doi.org/10.22456/1679-1916.15180

Carletto, A. \& Cambiaghi, S. (2018). Desenho Universal: um conceito para todos. São Paulo.

Manzini, E. \& Deliberato, D. (2006). Portal de ajudas técnicas para educação: equipamento e material pedagógico especial para educação, capacitação e recreação da pessoa com deficiência física: recursos para comunicação alternativa (2nd ed.). Brasília: MEC, SEESP.

Ministério da Saúde (2014). Diretrizes de atenção à pessoa com paralisia cerebral. Brasília: Ministério da Saúde.

Ribeiro, J. (2014). Heurísticas para avaliação da interação com aplicações (apps) de gráficos em dispositivos móveis touchscreen (Mestre). Universidade de Lisboa.

Simões, J. \& Bispo, R. (2006). Design inclusivo. Lisboa: Centro Português de Design.

Surveillance of Cerebral Palsy in Europe. (2017). SCPE. Retrieved 8 September 2017, from http://www.scpenetwork.eu/pt/my-scpe/manual-de-referencia-e-formacao/pc-esubtipos-de-pc/

World Health Organization, The World Bank (2012). Relatório mundial sobre a deficiência. São Paulo: SEDPcD.

Resumen: El presente artículo busca investigar las posibilidades de dispositivos touchscreen para el estímulo motor de personas con Parálisis Cerebral (PC) intentando contribuir a la accesibilidad de objetos de diseño a la más grande cantidad de usuarios conforme la premisa de Diseño Universal. Los procedimientos metodológicos empleados fueron revisión bibliográfica acerca de las tecnologías touchscreen y terapias para personas con Parálisis Cerebral y entrevistas semi estructuradas con profesionales de fisiatría, fisiotera- 
pia, fonoaudiología, terapia ocupacional y educación física con experiencia en la atención al público en cuestión, actuantes en el Centro Catarinense de Rehabilitación (CCR) de Florianópolis, Santa Catarina, en Brasil. Por la observación de los aspectos analizados, se concluyó que los dispositivos touchscreens se presentan como una herramienta a ser mejorada, ya que posibilitan una variedad de gestos manuales superior a otros dispositivos de tecnología asistiva computacional. El tamaño y la interfaz de las pantallas sensibles al tacto fueron algunos de los principales obstáculos identificados en la utilización de estas tecnologías por pacientes con Parálisis Cerebral. Es relevante, así, en el campo del Diseño Universal, explicar el cruce entre niveles de acometimiento motor de personas con PC y los gestos posibles en las pantallas, para contribuir al desarrollo de la accesibilidad en los dispositivos touchscreen no sólo a las personas con esa disfunción neurológica pero también a otras deficiencias motoras relacionadas.

Palabras clave: touchscreen - Parálisis Cerebral - Diseño Universal.

\begin{abstract}
This article aims to investigate the possibilities of touchscreen devices for motor stimulation of people with Cerebral Palsy (PC) in order to contribute to the accessibility of design objects to the greatest number of users according to the premise of Universal Design. The methodological procedures employed were a literature review on touchscreen technologies and therapies for people with Cerebral Palsy and semi-structured interviews with professionals in the area of physiatry, physiotherapy, speech therapy, occupational therapy and physical education who work in Centro Catarinense de Reabilitação (CCR) of Florianópolis, Santa Catarina, in Brazil, and have experience attending CP patients. By observing the aspects analyzed, it was concluded that touchscreen devices, with the due improvements, can be a tool in the rehabilitation process, since it enables a superior variety of manual movements than other devices of assistive technology. The size and interface of touchscreens are some of the major obstacles identified in the use of this technology by Cerebral Palsy patients. Therefore, it is relevant, in the field of Universal Design, to explore the intersection between the levels of motor involvement of people with $\mathrm{CP}$ and the possible gestures on the screens, so as to contribute to the advancement of digital accessibility not only to people with this neurological dysfunction but also several correlated motor impairments.
\end{abstract}

Key words: touchscreen - Cerebral Palsy - Universal Design. 